\title{
Smart-devices in Human Behavior Manipulation: Process diagram with exploratory assessment
}

\author{
Md. Mojibur Rahman Redoy Akanda*, Md. Alamgir Hossain \\ Department of Computer Science and Engineering, Prime University, Dhaka, Bangladesh. \\ *Corresponding author E-mail: alamgir.cse14.just@gmail.com
}

Manuscript received 15 April 2021; revised 1 May 2021; accepted 15 June 2021. Date of publication 3 July 2021

\begin{abstract}
Smart devices have become an essential part of human life with a bunch of modern features and facilities. Even in health care, health management, education, and the science sector use intelligent devices for their convenience. With the assertion of its wellness, people forget its downside and treating smart devices as their primary need. Whereas smart devices are tracking and collecting all user movements, including interest, boredom, and daily activity. As the data remain store in vendors' servers, and lightweight smart devices follow weak security, so data leakage also makes the data available to unauthorized parties. This sensitive data uses by vendors and third-party for business and various purposes to influence and manipulate human behavior by showing content mapping to the collected data. Because of the huge involvement of the user in smart-device, marketing strategy also changed a lot. Digital marketing has been introduced and become a key to success for many businesses where a particular content/advertisement can be mapped to particular leads. The next move of a user on the internet is shaping by applying numerous strategies based on previously collected data. In the era of smart devices, our personal life and personal data are not remaining personal anymore. This paper illustrates the systematic process of collecting and using data for manipulating human behavior. The raise of human behavior manipulation has been explained and an exploratory survey is imputed to strongly support the research statement.
\end{abstract}

Keywords: Behavior Manipulation, Controlling Human, Data Leakage, Smart-Device.

\section{Introduction}

The modern world is covered by technology and everyone avows its wellness. Smart devices playing a magnificent role in bringing technology into human's hands. Many features and facilities characterize those devices with a bunch of opportunities for a human to enjoy the world as a global village. Ease of use, internet connection, high speed, and a huge number of utility applications made them exceptionally attractive to people. With all these flairs, peoples are becoming highly dependent on it without any concern about its downside.

Under the study of Ofcom [1], it is noticed that most of the people in the UK are resting on digital devices and require an eternal internet connection. Their study also finds that $34 \%$ feel as cut off and $29 \%$ feel as lost if they do not get themselves online. The total number of smartphones user in the world is increased to 3.5 billion in 2020, which is going to reach 3.8 billion in 2021[2].

Wearable intelligent devices also becoming popular with peoples, they are using them for their simplicity to complete daily naive tasks. All the activity of humans can be monitored by those devices including physical and mental health (e.g. blood pressure, sleeping schedule, etc.) as it always remains connected with the human body. All the monitored data is stored on the vendor's server. The world's market for wearable devices has increased to $82.3 \%$ by shipping 118.9 million devices in the last 3 months of 2019 with a total of 336.5 million in the year [3].

With all these statistics, we can assume the rise of smart devices in this modern world and the user's fascination. As people are moving towards smart devices, so business also changed their marketing strategy from analog to digital marketing. Lower cost, global reach, and personalization made online marketing popular. By using modern marketing techniques, it is possible to show the information of products directly to a potential customer. Because humans all behavior is sensing by the smart devices and use this information to find the potential customer and then influence them about their product. Collecting human's data may have some advantages but it causes a large threat to human personal life. Information holders hold all the behavior of a particular person e.g., what is his/ her interest, boredom, hobby, sleeping time, working time, leisure time. With this information, it is possible to manipulate a person with various techniques. According to a report by Elisabeth Costa and David Halpern [4], people's behavior is impersonated and enumerated by the structure of the online environment.

This study will explain how smart devices collecting human data and controlling their behavior. Firstly, the rise of human behavior manipulation is explained and then the systematic operation of collecting and using data is delineated. Finally, an exploratory survey is explained. The survey explains the rate of human behavior controlling by smart devices and their impact on their regular social and online life. 


\section{Literature Review}

\subsection{The raise of human behavior manipulation}

Smartphones were first invented in 1992 and released in the marketplace for purchase in 1994 [5]. Personal computers were available before invented the internet. In 1989, Tim Berners-Lee designed the World Wide Web (WWW). At the end of 1990, the first web page served on the open web [6]. Smart devices are described by a few highlights including usability, speed, Internet association, just as download capacities. Smart device merchants are providing extensive support for clients by making accessible for them a wide variety of applications on their websites (Apps Stores) [7]. As indicated by datareportal [8], more than 4.57 billion individuals around the globe presently utilize the web - near 60 percent of the world's all-out population. At the beginning of 2020, more than 3.5 billion smartphone users are present worldwide. These huge amounts of clients are connected to the internet by using smartphones or computers [9]. When industries realize that, people all over the world are connecting to the internet, they start to develop marketing products online. In the meantime, there is a possibility to increase product quality through user reviews with a rating scale [10]. For getting new audiences, delivering different messages, and bring website traffic in, companies set different advertisements policy. It is called digital advertising or internet advertising. In 1994, the first banner advertisement had posted on a web page. After placing, the banner advertisement a new policy starts to analyze audience data and try to show advertisements to the target audience. In 1995, successful advertisements were placed on the target audience. For more efficient management of displaying advertisement campaigns across multiple websites and report on how users are interacting with advertisements, ROI (return on investment) tracking tool added in 1996 [11]. In 1997 advertisers introduced placed pop-up ads on the web page. At this point, the web was growing quickly and clients required a superior method to explore the terrain.

With search engines consistently picking up popularity, advertisers hoping to make advertisements that were more focused on and less accursed went to supported hunt as the following advanced publicizing frontier. That's way, advertisers turn to paid search and pay-perclick. It was running from 1999 to 2002. As social media platforms got steam during the 2000s, advertisers looked for an approach to coordinate advertisement content in a manner that was both successful and non-intrusive. Advertisers needed a strategy to arrive at more youthful web audiences who were progressively unswayed by banner ads and investing the vast majority of their web energy in social networks. This type of advertisement was starting in 2006. This is called Digital ads become hyper-targeted. From 2020 to the present, marketers finding value in native ads. Now they are working on behavioral targeting. By applying Artificial Intelligence, data mining, and deep learning, the pattern of user interests is generated. They also are working on predictive behavioral targeting. This is the process of inferring trends about audiences. For improving future advertisements, this analysis is used for particular users. For processing all types of advertisements, a huge amount of data is required from the audience for generating patterns by analysis. Ways of the data collection and processes are shown in the next section.

\subsection{The systematic process of collecting and using data for manipulation}

For controlling a user, it is necessary to be equipped with details information about that particular lead.

The type of information, amount of data, time frame, and methods need to be specific before collecting data. Basically, descriptive, behavioral, interaction, and attitudinal data are required to find out the characteristics of a particular lead. To collects, these types of data different sources are used. Web browsing, mobile app, in-store and e-commerce, subscription and registration, advertising platform, loyalty program, survey, social media, customer service, online sales department, hacking or unauthorized access, etc., data is used as a source.

A systematic process is shown in fig. 1 with its phase to provide a graphical clarification of collecting and using data for manipulation. In the first phase, it is illustrated that various smart devices (e.g. smartphones, tab, computers, smart TV, wearable devices, etc.,) play a vital role to collect data from users. Then the data organized in one platform. In the third phase, it determines an accurate adherence between the audience and smart devices. After gathering a sufficient amount of data, the analysis starts on the data by customer profiling and mapping user's journeys.
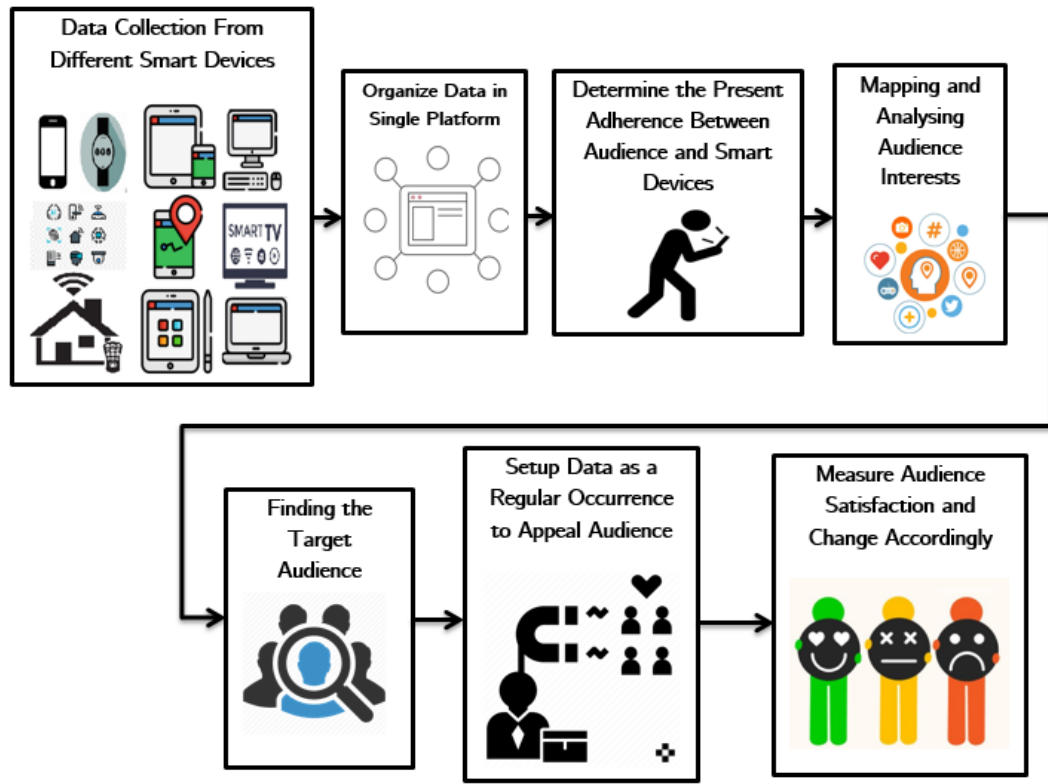

Fig 1. Systematic process of collecting and using data for manipulation

The analysis is continuing to improve the present audience and finding out the actual new audience, shown in phase 5. One important approach to incorporate bits of knowledge is personalization. Customize the connections with the clients, regardless of whether it is through an application, website, email, call, or different methods, by recollecting audience inclinations, offering a custom item or 
substance proposals, and tending to the audience by name. The present audience progressively anticipates these sorts of communications with the data collectors. To appeal to the audience with regular occurrence, data is set up for a particular audience. The information offers the opportunity to keep the measurements of the following audience experience and persistently upgrade the connection. This methodology sets up for ceaseless improvement and encourages all the effectively conform to changes in the audience's needs. Ensure each move is made to improve audience experience has an unmistakable goal, and that has an approach to quantify the outcomes. By including new estimation strategies, for example, short overviews toward the finish of client assistance call to measure fulfillment. As the following consequences of the endeavors to enhance the audience experience reveal possible regions of progress will come to plans and cycles. Roll out those improvements and keep observing measurements. As to continuing estimating and making a move, the audience experience will consistently improve. Basically, this audience satisfaction is running for a long time. During this whole time, the particular audience data is also processing for setting the actual interests. In this way, the placement of the audience's interests or features is implemented for a particular user to influence them.

\section{Methods}

Firstly, the rise of human behavior manipulation is delineated concerning the previous reports and empirical studies. The rise of online marketing and its important impact on the business revolution is explained. How they started to detect the target audience from the web and manipulate or influence them for a particular product is illustrated here.

Secondly, a systematic process diagram is illustrated to explain the user data collection process using smart devices and uses them to manipulate or influence people. Deep study and observation endorsed to identify the in a general systematic process.

Finally, a survey is circulated among peoples to know about their awareness knowledge and the impact of smart devices on behavior manipulation. The major question of this research is that the study tried to find the following answer: Are the peoples aware of data leakage by smart devices and the role of those data to manipulate human behavior?

\subsection{Demographics and magnitude of Smart-device use}

The first portion of this survey consists of questions regarding the participant's demographic information. Do questionnaires include gender, age, profession, organization/institute/company, and finally does the participant use the smartphone/laptop or not? The last question of this set has the value to determine if the participants move to the next set of questionnaires or not. Smartphones/laptops or holding both will move to the next section either submission of the survey will be completed with the no.

\subsection{Assessment of using Smartphones and Internet}

Completing the first section will direct the assessment to this segment. It is assumed to have a smartphone/ laptop or both for participants contributing to this section. Smartphones use since the year, internet uses since the year, daily internet uses (in hours), and the purpose of using the internet considered in this part.

\subsection{Uses of application software and awareness about personal data}

In this segment, reading terms, conditions, and allowing permission while installing software is asked in this part. The survey introduces a list in the first question with numerous popular application software (Facebook, Messenger, etc., and others for user entry) to find the most uses application software. The reliability of having smartphones in life is also queried.

\subsection{Behavior manipulation occurrences}

The questionnaires of this segment are the devoted focus of this research. Questionnaires are prepared to measure the incidences of behavior manipulation. Knowledge of data collection from users using spying by smart devices, search something over the internet and observe related advertisements approaching home, frequency of clicking those advertisements, etc. taken into consideration in this section.

\subsection{Procedure}

The survey was designed in Google form. The link of the survey is circulated to social media following the chain-sampling method. Email is also used to send the link to specific peoples. Without having smart devices respondents cannot participate in the survey. Each section has a clear explanation for participants so that they can answer it easily. The nature of the study was explained to respondents with debriefing discussions.

\subsection{Participants}

A total of 259 participants responds against our investigation and 5 of them have exonerated for vogue information. Among 254 respondents, male $72.33 \%$ and $27.67 \%$ of females are detected with their ages between 20 and 25 , which was three quarters. Students were the major part of this study with their cordial response to the form, 77.87 percent of students attended from universities, colleges. Rests of the participants were engineers, doctors, teachers, service holders, and businessperson.

\section{Results and Discussion}

Among various smart devices, more than half of the participants use both smartphones and laptops. The majority of respondents started to use smartphones before 2017 and the rate is 88.10 percent. Samely, the participants of the study started using the internet in the early period of the decade and above the quarter of their daily internet uses duration is more than 10 hours. These data assure to consider the participants of the study as strong users of smart devices and the internet. 


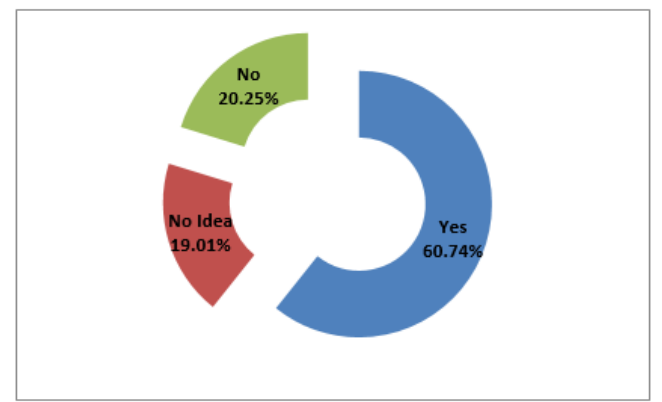

Fig 2. Knowledge about spying

Between this sound user, the study found that about a quarter of respondents do not read terms and conditions before installing application software and about twelve and a half percent do not have any idea about this. Similarly, 27.27 percent of participants do not lookups before allowing permission, and 8.68 percent have no idea about it. Whereas installing software without reading terms, conditions, and allowing permission is a venal issue for data leakage.

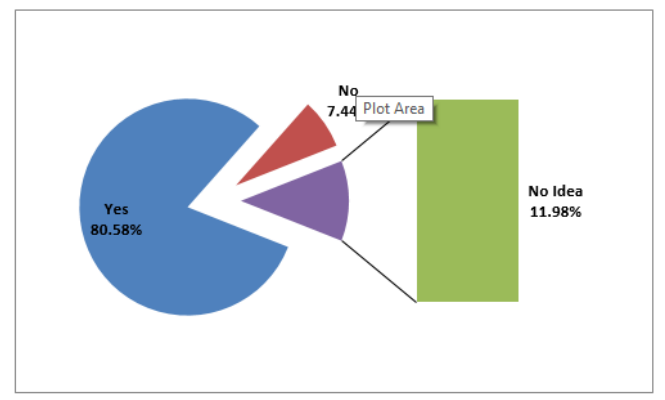

Fig 3. Showing advertisement based on searching

The majority of participants with the rate of 88.43 percent believe smart devices are making people's life reliable but very few amounts with 11.57 percent do not think so. Figure 2 delineates the knowledge of respondents about spying using smart devices. The chart shows that around 20 percent do know nothing about spying and 19.01\% do not have any idea about it. So, combinedly it is assumed that just above 39 percent of peoples do not have an idea about spying using smart devices. As per the previous study of this research, the search history of the user is a data set uses for customer profiling. In figure 3, it is illustrated that more than 80 percent of participants face the issue of getting relevant commercials based on their previous searching history. Just about 12 percent respond with no idea and a 7.44 percent reply, as they did not face the scenario. This study observed that the commercial able to influence 47.93 percent of respondents and the rest of them are capable to ignore it.

Smart devices are making people's life reliable; the majority of participants believe this with a rate of 88.43 percent but very few amounts with 11.57 percent do not. Figure 2 delineates the knowledge of respondents about spying using smart devices. The chart shows that around 20 percent do know nothing about spying and $19.01 \%$ do not have any idea about it. So, combinedly it is assumed that just above 39 percent of peoples do not have an idea about spying using smart devices. As per the previous study of this research, searching the history of the user is such a data set uses for customer profiling. In figure 3, it is illustrated that more than 80 percent of participants face the issue of getting relevant commercials based on their previous searching history. Just about 12 percent respond back with no idea and a 7.44 percent reply, as they did not face the scenario. This study observed that the commercial able to influence 47.93 percent of respondents and the rest of them are capable to ignore it.

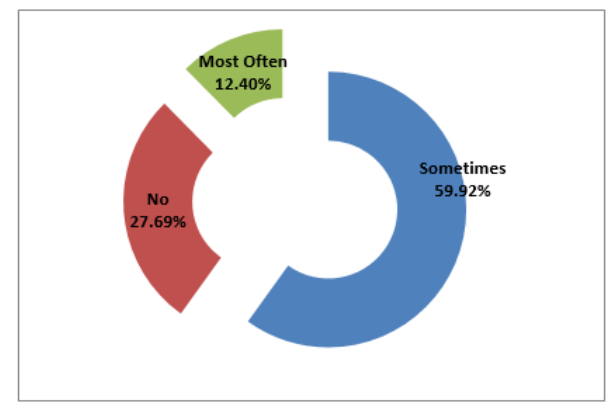

Fig 4. Rate of clicking on the commercial

Figure 4 has shown the rate of clicking on advertisements after appearing on the screen. Just above quarters of participants ignore clicking those commercials and surprisingly among the rest of the percentage, almost twelve and a half click them most often and around sixty percent sometimes click on the commercial. 


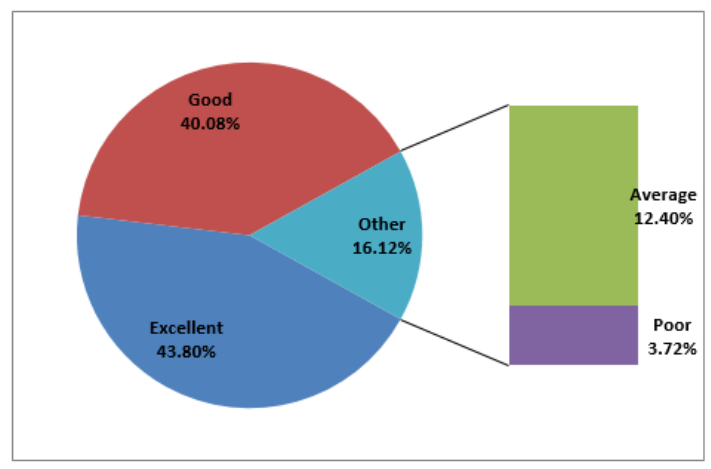

Fig 5. Participant's feedback about this survey

People's active with other tasks try to know time from the phone and after a little, they observe hours have gone on it, where they become busy with other features of the phone delivered to manipulate them. Unexpectedly, 26.14 percent of peoples faced this most often and 63.49 percent faced the issue sometimes.

Social media platforms become popular in this decade for communication. Peoples from different sections post various contents on social media and users provide react regarding their interest in the content. This type of data is also used for customer profiling. As a result, some type of content appears on those platforms for a particular user. This study found 78.93 percent of respondents observed the same scenario while using social media.

Around three of the quarter participants of this exploratory study believe they are passing maximum leisure time with smart devices for their features instead of passing with family. On the other hand, 83.06 percent confess that smart device creating an impact on their social life.

This study also collects feedback from participants about the survey experience. In fig.5 the outcomes of respondents have shown. Almost 44 percent marked it as excellent and about 40 percent as good. Very few amounts $(3.72 \%)$ of people marked this as poor whether $12.40 \%$ believe the quality is average.

\section{Conclusions}

Smart devices have shown their application and continuing their enlargement to allure users. Ease of use makes it comfortable for all age users whether they may not have any idea regarding data privacy. Human behavior manipulation occurs by the data collecting from smart devices. People's personal information is becoming public these days and personal life is not lasting personal any longer.

It became mandatory for users to read terms and conditions before taking any services and vendors need to stop their spying on users. The researcher needs a high intention to develop such an intelligent system for smart devices, which will block all personal data leakage from the devices. Finally, smart devices need to continue their enhancement for the demand of the modern world without suffering any undesirable belongings.

\section{Acknowledgement}

The authors express their appreciation to the colleagues who reviewed this paper and offered useful recommendations for improving it. The authors greatly appreciate the time and useful suggestions from guest editors and reviewers.

\section{References}

[1] Ofcom. A decade of digital dependency. 2018 [cited 2020 07]; Available from: https://www.ofcom.org.uk/aboutofcom/latest/features-and-news/decade-of-digital-dependency.

[2] Statista, Number of smartphone users worldwide from 2016 to 2021. 2020.

[3] IDC, Shipments of wearable devices reach 118.9 million units in the fourth quarter and 336.5 million for 2019. 2020.

[4] Elisabeth Costa, D.H., The behavioural science of online harm and manipulation, and what to do about it. The Behavioural Insights Team, 2019.

[5] Tocci, M. History and evolution of smartphones. 2020 [cited 2020 10/10]; Available from: https://simpletexting.com/where-havewe-come-since-the-first-smartphone/.

[6] Tocci, M. History of the web. 2020 [cited 2020 20/11]; Available from: https://webfoundation.org/about/vision/history-of-the-web/.

[7] AW WAI YAN, K.M.-N., EMAD ABU-SHANAB, JANEJIRA SUTANONPAIBOON, Factors that Affect Mobile Telephone Users to Use Mobile Payment Solution. Int. Journal of Economics and Management, 2009. 3(1): p. 37-49.

[8] DATAREPORTAL, DIGITAL AROUND THE WORLD. 2020.

[9] Miva, The History Of Ecommerce: How Did It All Begin? 2011.

[10] Reed, D. History of Online Advertising. 2017 [cited 2020 25/12]; Available from: https://study.com/academy/lesson/history-ofonline-advertising.html.

[11] Cook, K., A Brief History of Online Advertising, in HubSpot. 2016. 